\title{
Systematic assessment of obesity information on the microblogging platform Twitter
}

\author{
C.H. Ellis, C.E.L. Evans and J.B. Moore \\ School of Food Science and Nutrition, University of Leeds, Leeds, LS2 9JT
}

Previous research has demonstrated that media reporting concerning obesity is poorer quality and involves stigmatising discourse ${ }^{(1)}$. The microblogging social media platform, Twitter, has 321 million users sharing information globally and is therefore of particular research interest. This study aimed to use web scraping and linguistic techniques to collect and analyse the quality of obesity information on Twitter.

Orgneat, a .net based project using Twitter scraping principals ${ }^{(2)}$, was used to download tweets and associated engagement data posted from 00:01-23:59 on 30 August 2019 that included the terms 'obesity' or '\#obesity'. Tweets were screened to remove duplicates and to identify those containing uniform resource locators (URLs), deemed more likely to be disseminating information from an original source rather than opinion. $20 \%$ of the clean data were randomly selected for analysis in Excel. Each URL was reviewed manually and categorised based on the website source and the content before being assessed against 10 quality criteria, adapted from research assessing the quality of nutrition reporting in the media ${ }^{(3)}$.

During the selected 24-hour period, 1726 tweets including either obesity or \#obesity were returned, 818 of these contained a URL. After manually cleaning the data, $n=636$ unique URLs remained and $20 \%$ were randomly selected for qualitative analysis $(n=128)$. The URLs shared were categorised based on content. As the focus was on blogs and news media, 93 URLs were excluded because they linked to scientific research papers $(n=21)$, duplicate articles $(n=14)$, paid content $(n=21)$, advertising $(n=11)$ or irrelevant content (recipes, legal cases or government guidelines, $n=26$ ). The remaining $n=27$ blogs, $n=5$ news articles, and $n=3$ press releases, were scored positively or negatively against the 10 quality criteria. Of these, press releases were found to have the highest quality reporting with $67 \%$ of articles analysed scoring +6 or higher. While $60 \%$ of news articles analysed scored +6 or above, blogs were found to have the lowest quality reporting; with only $41 \%$ of blogs scoring +6 or above. However, blogs received significantly more engagement on Twitter through likes, retweets and comments than the other content analysed with 115 total interactions (an average of 4.26/blog). In contrast, press releases received the lowest engagement with only two interactions recorded, an average of $0.66 /$ press release.

This investigation of the quality of obesity information shared on Twitter suggests that blogs, in contrast to news articles and press releases, have the lowest quality reporting but receive the greatest engagement. Although sample size and the disproportionate number of articles categorised as blogs are current limitations of this pilot survey; this work provides the first transdisciplinary process to collect, analyse, and interpret obesity-related posts on Twitter.

1. Brooker P, Barnett J, Vines J, et al., (2018) New Media \& Society 20, 3201-3222

2. OrgneatUI. vol. 2019. https://github.com/rahulha/OrgneatUI accessed August 2019

3. Kininmonth A, Jamil N, Almatrouk N, et al. (2017) BMJ Open 7 